\title{
HIGH FREQUENCY DIFFRACTION OF CYLINDRICAL WAVES BY PERFECTLY CONDUCTING SUCCESSIVE STEP DISCONTINUITIES
}

\author{
$\underline{\text { B. Türetken }^{(1)}}$, A. Alkumru ${ }^{(2)}$, A. Büyükaksoy ${ }^{(3)} \quad$ A. Kuştepeli $^{(4)}$ \\ (1) The Scientific and Technological Research Council of Turkey \\ National Research Institute of Electronics and Cryptology 41470, Gebze, Kocaeli, Turkey \\ E-mail: bahattin@,uekae.tubitak.gov.tr \\ (2) Gebze Institute of Technology, Department of Electronics Engineering \\ 41400, Gebze, Kocaeli, Turkey \\ (3) Gebze Institute of Technology, Department of Mathematics \\ 41400, Gebze, Kocaeli, Turkey \\ (4) İzmir Institute of Technology, Department of Electrical and Electronics Engineering \\ Gülbahçe Köyü, Urla, İzmir, Turkey
}

\begin{abstract}
The diffraction of high frequency cylindrical electromagnetic waves by step discontinuities is investigated rigorously by using the Fourier transform technique in conjunction with the mode matching method. The hybrid method of formulation gives rise to a scalar Wiener-Hopf equation of the third kind, the solution of which contains infinitely many constants satisfying infinite systems of linear algebraic equations.
\end{abstract}

\section{INTRODUCTION}

The problem of diffraction of high frequency electromagnetic waves by step discontinuities constitutes one of the important topics in diffraction theory and is relevant for many engineering applications. This kind of a problem is first considered by Johansen [1] in the case of a surface wave diffraction by a reactive step which joins two reactive half-planes. The scattering problems considered by Büyükaksoy and Birbir [2-4], Büyükaksoy and Tayyar [5] and Tayyar, Aksoy and Alkumru [6] can also be cited for the diffraction of plane, cylindrical and surface waves by different impedance step discontinuities, respectively.

The aim of this work is to obtain a rigorous solution for the diffraction problem of cylindrical waves by perfectly conducting successive step discontinuities. For this purpose the step geometry given in the Figure-1 is illuminated by a time harmonic line source located along an infinitely long straight line $\mathrm{S}$ which is parallel to the $\mathrm{z}$-axis. By introducing the Fourier transform for the scattered field and applying the related boundary conditions in the transform domain, the problem is reduced into a Modified Wiener-Hopf Equation (MWHE) of the third kind. The solution of this MWHE involves two types of branch-cut integrals and five sets of infinitely many constants satisfying five infinite systems of linear algebraic equations. One type of branch-cut integrals can be evaluated approximately for $k l>>1$ while a numerical treatment is required for the other type. A time factor $\exp (-i \omega t)$ is assumed and suppressed throughout the paper.

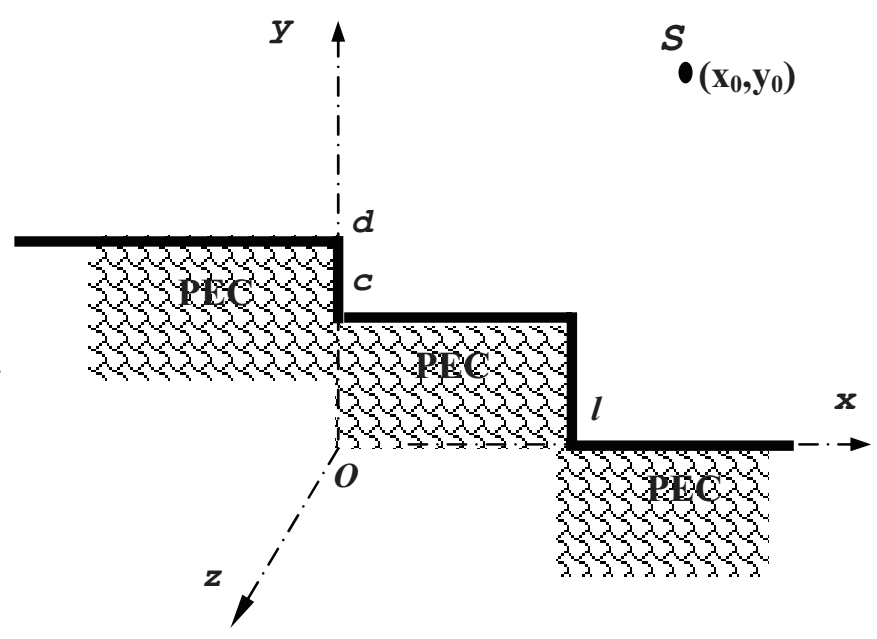

Fig.1. Geometry of the diffraction problem 


\section{FORMULATION AND SOLUTION OF THE PROBLEM}

Consider the diffraction of a line source $\mathrm{S}$ by perfectly step discontinuities defined by $\{\mathrm{y}=\mathrm{d}, \mathrm{x}(-\infty, 0)\} \mathrm{U}$ $\{\mathrm{y} \in(\mathrm{c}, \mathrm{d}), \mathrm{x}=0\} \mathrm{U}\{\mathrm{y}=\mathrm{c}, \mathrm{x} \in(0,1)\} \quad \mathrm{U}\{\mathrm{y} \in(0, \mathrm{c}), \mathrm{x}=1\} \quad \mathrm{U}\{\mathrm{y}=0, \mathrm{x} \in(1, \infty)\}$ where $(\mathrm{x}, \mathrm{y})$ denote the usual cartesian coordinates (see Figure 1). The current density associated with this line source is represented by

$$
\vec{J}=I \delta\left(x-x_{0}\right) \delta\left(y-y_{0}\right) \vec{e}_{z}
$$

and the total electric field which is parallel to the z-axis can also be written as

$$
\vec{E}(x, y)=u(x, y) \vec{e}_{z} .
$$

In (1) $I$ stands for the strength of the exciting source, $\vec{e}_{z}$ shows the unit vector of the z-axis and $\delta($.$) is the$ standart Dirac distribution function while in (2) $u(x, y)$ which satisfies the equation

$$
\Delta u+k^{2} u=-i \omega \mu I \delta\left(x-x_{0}\right) \delta\left(y-y_{0}\right)
$$

is the expression to be determined with the help of well known boundary , edge and radiation conditions for the perfectly conducting structures. In (3) $k$ is the free space wave number having a small positive imaginary part which will tend to zero at the end of the analysis. By taking the Fourier transform of $u(x, y)$ with respect to $\mathrm{x}$ variable and considering also above mentioned boundary and continuity conditions in the transform domain $\alpha$, the problem is reduced into a following Modified Wiener-Hopf Equation of the third kind which is valid in the strip $\operatorname{Im}(-k)<\operatorname{Im}(\alpha)<\operatorname{Im}(k)$ :

$$
\frac{G_{1}(\alpha, d)}{M(\alpha)}+e^{i \alpha l} \frac{G_{+}(\alpha, d)}{N(\alpha)}+\dot{G}_{-}(\alpha, d)=-2 i K(\alpha) C(\alpha)+\sum_{n=1}^{\infty}(-1)^{n}\left\{\frac{K_{n} f_{n}}{\left(\alpha^{2}-\alpha_{n}^{2}\right)}+e^{i \alpha l}\left[\gamma_{n} \frac{\left\{m_{n}-i \alpha n_{n}\right\}}{\left(\alpha^{2}-\beta_{n}^{2}\right)}-K_{n} \frac{\left\{g_{n}-i \alpha h_{n}\right\}}{\left(\alpha^{2}-\alpha_{n}^{2}\right)}\right]\right\}
$$

with

$$
\begin{gathered}
M(\alpha)=\frac{\sin [K(\alpha)(d-c)]}{K(\alpha)} e^{i K(\alpha)(d-c)} \quad, \quad N(\alpha)=\frac{\sin [K(\alpha) d]}{K(\alpha)} e^{i K(\alpha) d} \\
\alpha_{n}=k \sqrt{1-\left[\frac{n \pi}{k(d-c)}\right]^{2}}, \quad K_{n}=\frac{n \pi}{d-c} \quad, \quad \beta_{n}=k \sqrt{1-\left(\frac{n \pi}{k d}\right)^{2}} \quad, \quad \gamma_{n}=\frac{n \pi}{d} \quad, \quad n=0,1,2, \ldots . .
\end{gathered}
$$

and

$$
C(\alpha)=\frac{k Z I e^{i \alpha x_{0}} e^{i K(\alpha)\left(y_{0}-d\right)}}{2 K(\alpha)}
$$

where $\mathrm{Z}$ is the characteristic impedance of the free space. $K(\alpha)$ appearing in the above expressions stands for the square-root function $K(\alpha)=\sqrt{\alpha^{2}-k^{2}}$ defined in the complex $\alpha$-plane cut along $(-\infty,-k) \cup(k, \infty)$ with the condition $K(0)=-i k$. In (4) $G_{1}(\alpha, d)$ is an entire function while $G_{+}(\alpha, d)$ and $\dot{G}_{-}(\alpha, d)$ are the regular functions of $\alpha$ in the upper $\operatorname{Im}(\alpha)>-k$ and lower $\operatorname{Im}(\alpha)<k$ half-planes, respectively.

By using the factorization and decomposition procedures together with the consideration of the edge conditions which are required for the application of the Liouville theorem, the Modified Wiener-Hopf Equation in (4) can be reduced to the following coupled system of Fredholm integral equations of the second kind:

$$
M_{-}(\alpha) R(\alpha)=\frac{1}{2 \pi i} \int_{L^{-}} M_{-}(\tau) S(\tau) \frac{e^{i \tau l}}{(\tau-\alpha)} d \tau+\frac{1}{\pi} \int_{L^{-}} \frac{K(\tau) C(\tau) M_{-}(\tau)}{(\tau-\alpha)} d \tau+\sum_{n=1}^{\infty} \frac{(-1)^{n} K_{n} f_{n} M_{+}\left(\alpha_{n}\right)}{2 \alpha_{n}\left(\alpha+\alpha_{n}\right)}
$$

and

$$
\begin{aligned}
M_{+}(\alpha) N_{-}(\alpha) S(\alpha)=-\frac{1}{2 \pi i} \int_{L^{+}} M_{+}(\tau) N_{-}(\tau) R(\tau) \frac{e^{-i \tau l}}{(\tau-\alpha)} d \tau & -\frac{1}{\pi} \int_{L^{+}} \frac{K(\tau) C(\tau) M_{+}(\tau) N_{-}}{(\tau-\alpha)} e^{-i \tau l} d \tau \\
& +\sum_{n=1}^{\infty} \frac{(-1)^{n} K_{n}\left(g_{n}-i \alpha_{n} h_{n}\right) M_{+}\left(\alpha_{n}\right) N_{-}\left(\alpha_{n}\right)}{2 \alpha_{n}\left(\alpha+\alpha_{n}\right)}+J(\alpha)
\end{aligned}
$$


where

$$
R(\alpha)=\dot{G}_{-}(\alpha, d)-\sum_{n=1}^{\infty} \frac{(-1)^{n} K_{n} f_{n}}{\left(\alpha^{2}-\alpha_{n}^{2}\right)} \quad, \quad S(\alpha)=\frac{G_{+}(\alpha, d)}{N(\alpha)}+\sum_{n=1}^{\infty}(-1)^{n}\left[\frac{K_{n}\left(g_{n}-i \alpha h_{n}\right)}{\left(\alpha^{2}-\alpha_{n}^{2}\right)}-\frac{\gamma_{n}\left(m_{n}-i \alpha n_{n}\right)}{\left(\alpha^{2}-\beta_{n}^{2}\right)}\right]
$$

and

$$
J(\alpha)=-\frac{1}{\pi} \int_{C^{+}} \frac{M_{+}(\tau)}{N_{+}(\tau)} \frac{\sin ^{2}[K(\tau) d]}{K(\tau)}\left\{\sum_{n=1}^{\infty}(-1)^{n}\left[\frac{K_{n}\left(g_{n}-i \alpha h_{n}\right)}{\left(\alpha^{2}-\alpha_{n}^{2}\right)}-\frac{\gamma_{n}\left(m_{n}-i \alpha n_{n}\right)}{\left(\alpha^{2}-\beta_{n}^{2}\right)}\right]\right\} \frac{d \tau}{(\tau-\alpha)} .
$$

In $(8 \mathrm{a}, \mathrm{b})$ the functions $M_{ \pm}(\alpha)$ and $N_{ \pm}(\alpha)$ are defined through $M(\alpha)=M_{+}(\alpha) M_{-}(\alpha)$ and $N(\alpha)=N_{+}(\alpha) N_{-}(\alpha)$.

$M_{+}(\alpha)$ and $N_{+}(\alpha)$ are regular and free of zero in the upper half-plane while $M_{-}(\alpha)$ and $N_{-}(\alpha)$ have the same properties in the lower half-plane. The explicit expressions of these ( \pm ) functions are given in [7].

For $\mathrm{kl}>>1$ the solution of the coupled system of Fredholm integral equations in $(8 \mathrm{a}, \mathrm{b})$ can be obtained by iterations as

$$
R(\alpha)=\sum_{n=1}^{\infty} R^{(j)}(\alpha)=\frac{1}{M_{-}(\alpha)} \sum_{n=1}^{\infty} P^{(j)}(\alpha) \quad, \quad S(\alpha)=\sum_{n=1}^{\infty} S^{(j)}(\alpha)=\frac{1}{M_{+}(\alpha) N_{-}(\alpha)} \sum_{n=1}^{\infty} Q^{(j)}(\alpha) .
$$

The unknown expansion coefficients $f_{n}, g_{n}, h_{n}, m_{n}$ and $n_{n}$ appearing in $(8 \mathrm{a}, \mathrm{b})$ and $(9 \mathrm{a}, \mathrm{b}, \mathrm{c})$ can also be determined approximately from the consideration of the infinite systems of linear algebraic equations given by

$$
\begin{array}{rrr}
f_{n} \quad(-1)^{n} \frac{2 n \pi}{(d-c)^{2}} M_{+}\left(\alpha_{n}\right)\left[P^{(1)}\left(\alpha_{n}\right)+P^{(2)}\left(\alpha_{n}\right)\right], \quad g_{n}+i \alpha_{n} h_{n} \quad(-1)^{n+1} \frac{2 n \pi}{(d-c)^{2}} \frac{M_{+}\left(\alpha_{n}\right)}{N_{+}\left(\alpha_{n}\right)}\left[Q^{(1)}\left(-\alpha_{n}\right)+Q^{(2)}\left(-\alpha_{n}\right)\right] \\
n=1,2, \ldots \quad(11 \mathrm{a}, \mathrm{b})
\end{array}
$$

and

$$
Q^{(1)}\left(\beta_{n}\right) \quad-Q^{(2)}\left(-\beta_{n}\right) \quad, \quad n_{n}=\frac{2 \sin \left(\gamma_{n} c\right)}{d} \sum_{m=1}^{\infty} \frac{K_{m}}{\left(K_{m}^{2}-\gamma_{n}^{2}\right)} h_{m}, \quad g_{n}=\frac{2 K_{n}}{(d-c)} \sum_{m=1}^{\infty} \frac{\sin \left(\gamma_{m} c\right)}{\left(K_{n}^{2}-\gamma_{m}^{2}\right)} m_{m}, n=1,2, . . \quad(11 \mathrm{c}, \mathrm{d}, \mathrm{e})
$$

Since all the unknown constants are determined according to this result, the functions $G_{1}(\alpha, d), G_{+}(\alpha, d)$ and $\dot{G}_{-}(\alpha, d)$ can also be obtained. So, the total field which is valid for the whole space can easily be computed by taking inverse Fourier Transform of the spectral coefficient.

\section{REFERENCES}

[1] E. L. Johansen, "Surface wave scattering by a step" IEEE Trans. Antennas and Propagat. AP-15, 442-448, 1967.

[2] A. Büyükaksoy and F. Birbir, "Plane wave diffraction by an impedance step" IEEE Trans. Antennas and Propagat., AP-41, 1160-1164, 1993.

[3] A. Büyükaksoy and F. Birbir, "Correction to plane wave diffraction by an impedance step" IEEE Trans. Antennas and Propagat. AP-44, 422, 1996.

[4] F. Birbir and A. Büyükaksoy, "Plane wave diffraction by a reactive step" Int. J.Engng. Sci. Vol. 35, 311$319,1997$.

[5] A. Büyükaksoy and I.H. Tayyar, " High frequency diffraction by a rectangular impedance cylinder on an impedance plane" IEE Proc. Sci. Meas. Technol. Vol.149, 49-59, 2002.

[6] I.H. Tayyar, S. Aksoy and A. Alkumru, "Surface wave scattering by a rectangular impedance cylinder located on a reactive plane" Math. Meth. Appl. Sci. Vol. 28, 525-549, 2005.

[7] B. Noble, "Methods based on the Wiener-Hopf Technique", Pergamon Press, New York, 1958. 